\title{
Clinical utility of tumor mutational burden in patients with non- small cell lung cancer treated with immunotherapy
}

\author{
Lizza E. Hendriks ${ }^{1,2}$, Etienne Rouleau ${ }^{3}$, Benjamin Besse ${ }^{1,4}$ \\ ${ }^{1}$ Department of Cancer Medicine, Gustave Roussy, Institut d'Oncologie Thoracique (IOT), Gustave Roussy, Villejuif, France; ${ }^{2}$ Department \\ of Pulmonary Diseases, GROW - School for Oncology and Developmental Biology, Maastricht University Medical Center, Maastricht, The \\ Netherlands; ${ }^{3}$ Department of Medical Biology and Pathology, Gustave Roussy, Cancer Genetics Laboratory, Gustave Roussy, Villejuif, France; \\ ${ }^{4}$ Paris-Sud University, Orsay, France \\ Contributions: (I) Conception and design: All authors; (II) Administrative support: None; (III) Provision of study materials or patients: None; (IV) \\ Collection and assembly of data: LE Hendriks, E Rouleau; (V) Data analysis and interpretation: All authors; (VI) Manuscript writing: All authors; (VII) \\ Final approval of manuscript: All authors. \\ Correspondence to: Lizza E. Hendriks, MD, PhD. Department of Pulmonary Diseases, GROW - School for Oncology and Developmental Biology, \\ Maastricht University Medical Center, PO Box 5800, 6202 AZ, Maastricht, The Netherlands. Email: lizza.hendriks@mumc.nl.
}

\begin{abstract}
Anti-programmed death (ligand)-1 [anti-PD-(L)1] therapies such as pembrolizumab, nivolumab or atezolizumab have become standard of care for non-small cell lung cancer (NSCLC) patients either in first line or beyond. PD-L1 expression level allows enriching the treated population with responders, but it is still not an optimal biomarker. Even in patients with PD-L1 tumor proportion score (TPS) levels of $\geq 50 \%$ treated with first line pembrolizumab overall response rate (ORR) is only $44.8 \%$ and overall survival at one year is $70 \%$. Moreover, in combination trials with chemotherapy and anti-PD-(L)1 therapy, a significant proportion of patients does not respond (ORR ranges from 45.3\% to 64.0\%), regardless of PD-L1 expression. Furthermore, PD-L1 expression level is not associated with improved benefit in patients treated with combinations of anti-PD-(L)1 and anti-cytotoxic T-lymphocyte-associated antigen (anti-CTLA4) therapy. One of the new promising biomarkers is tumor mutational burden (TMB). It has been discovered that especially tumor types with a known high mutation rate such as NSCLC and melanoma respond best to immune checkpoint inhibitors (ICIs). An explanation is that this high mutation rate makes it more likely that neoantigens arise that are targeted by activated immune cells, but it is not feasible to determine neoantigen load in daily practice. However, TMB of a certain tumor type is associated with neoantigen load and outcome on ICIs. In this comprehensive review, we discuss the TMB analysis methods, the rationale to use TMB as a predictive biomarker and the clinical utility of TMB in NSCLC patients treated with ICIs.
\end{abstract}

Keywords: Immunotherapy; mutation/immunology; non-small cell lung cancer (NSCLC); predictive value of tests; programmed death-ligand 1 (PD-L1)

Submitted May 08, 2018. Accepted for publication Sep 25, 2018.

doi: 10.21037/tlcr.2018.09.22

View this article at: http://dx.doi.org/10.21037/tlcr.2018.09.22

\section{Introduction}

In less than a decade, immune checkpoint inhibitors (ICIs) have become standard of care in the treatment of advanced non-small cell lung cancer (NSCLC), either in first line (alone in selected patients or in the near future in combination in others) or beyond. Pembrolizumab, a programmed death-1 (PD-1) inhibitor is registered in first line for advanced NSCLC patients, without an activating epidermal growth factor receptor $(E G F R)$ mutation or anaplastic lymphoma kinase $(A L K)$ aberration, but with a programmed death-ligand 1 (PD-L1) tumor proportion score (TPS) of $\geq 50 \%$. It is also registered in second line for those with a PD-L1 TPS of $\geq 1 \%$. This 
registration is based on the results of the KEYNOTE-024 (pembrolizumab versus platinum-doublet chemotherapy) and KEYNOTE-010 (pembrolizumab versus docetaxel) trials, respectively $(1,2)$. In second line, nivolumab, another PD-1 inhibitor, is also registered regardless of PD-L1 expression based on the results of the CheckMate 017 trial (squamous NSCLC, nivolumab versus docetaxel) and CheckMate 057 trial (non-squamous NSCLC, nivolumab versus docetaxel) $(3,4)$. Atezolizumab, a PD-L1 inhibitor, is also registered for second line treatment regardless of PD-L1 expression based on the results of the OAK trial (atezolizumab versus docetaxel) (5). Although PDL1 expression is a predictive biomarker for outcome on PD-(L) 1 inhibitor, it is not a perfect biomarker as even in the patients with a PD-L1 expression of $\geq 50 \%$ the overall response rate (ORR) is only $44.8 \%$ (1). Moreover, PD-L1 expression level did not adequately enrich the first line population to demonstrate that single agent nivolumab is superior to platinum-doublet chemotherapy. In the CheckMate 026 trial, untreated advanced NSCLC with a PD-L1 TPS of $\geq 1 \%$ were randomized between nivolumab and platinum-doublet chemotherapy, primary endpoint was PFS among patients with a PD-L1 expression level of $\geq 5 \%$. The trial was negative for its primary endpoint, but also in the exploratory subgroup analysis of patients with a PD-L1 TPS of $\geq 50 \%$ no benefit of nivolumab over chemotherapy was seen (6). Furthermore, in trials with combination ICI such as the phase II CheckMate 568 [nivolumab combined with ipilimumab, a cytotoxic T-lymphocyte-associated antigen (CTLA-4) antibody], the randomized phase III CheckMate 227 trial (nivolumab plus ipilimumab versus chemotherapy or nivolumab monotherapy) and the phase Ib trial of durvalumab (anti-PD-L1) and tremelimumab (anti-CTLA4), ICI benefit was seen irrespective of PDL1 expression level (7-9). Last, in the randomized phase III KEYNOTE-189 trial (first line platinum doublet chemotherapy with pembrolizumab or placebo in nonsquamous NSCLC) and the randomized phase III IMPOWER 150 trial (carboplatin/paclitaxel combined with either atezolizumab, atezolizumab/bevacizumab or bevacizumab) a benefit of adding PD-(L)1 inhibition to chemotherapy was seen regardless of PD-L1 expression level, although benefit was highest in those with high PDL1 status $(10,11)$. As even in these combination therapy trials a significant proportion of patient did not respond (ORR ranged from $45.3 \%$ to $64.0 \%$ ) and not all responding patients had long-term benefit $(7,10,11)$, other biomarkers are needed that are better able to predict beneficial outcome of ICI treatment. Until now, besides PD-L1, only mismatch repair (MMR) deficiency has been Food and Drug Administration (FDA) approved as a predictive biomarker (12). Moreover, there are several issues on PDL1 testing such as the use of different antibodies, different platforms and different cut-offs for positivity that are extensively reviewed elsewhere (13).

One of the new promising biomarkers is tumor mutational burden (TMB). In this comprehensive review, we discuss the rationale to use TMB as a predictive biomarker, the analysis methods and the clinical utility of TMB in NSCLC patients treated with ICI.

\section{TMB definition and TMB assessment methods}

TMB has first been determined with whole exome sequencing (WES). Currently, WES seems to be less feasible in daily practice as it is costly, time consuming, labour intensive and needs large sequencing capacity $(14,15)$. TMB can also be determined through hybrid-capture based targeted next generation sequencing [large next-generation sequencing (NGS) panel-NGS laboratory developed test (LDT)], for example using the FoundationOne ${ }^{\circledR}$ assay (first version of $\mathrm{F} 1 \mathrm{LDT}$ test) or the FoundationOne $\mathrm{CDx}{ }^{\mathrm{TM}}$ assay (F1 CDx ${ }^{\mathrm{TM}}$, current FoundationOne assay) (16-18). In contrast to WES, large panel NGS is increasingly used in daily practice, usually with a limited number of genes. TMB is defined as the total number of somatic mutations in the tumor exome (17). There are different ways to estimate the total number of somatic mutations. The parameters are the type of mutations, the minimal allelic frequency and the strategy of excluding germline variants. In WES, the germline variants are oftenly excluded with a WES applied to healthy tissue. The results are expressed as a total number of mutations. The NGS FoundationOne assays define TMB as the total number of synonymous and nonsynonymous mutations present at $\geq 5 \%$ allele frequency (after filtering) in the sequenced tumor genome. Results are expressed as mut/Mb $(18,19)$. Multiple platforms exist for large NGS panel analyses. Some assays have correlated the TMB result from WES to a large NGS panel. For example, the F1 LDT assay with a region of interest around 1.1 megabase $(\mathrm{Mb})$ and 315 genes has been correlated to WES (16), with a Spearman's $r=0.9$, and positive and negative agreements between methods both $86 \%$ (20). Furthermore, TMB values of the F1 LDT and the $\mathrm{F} 1 \mathrm{CDx}^{\mathrm{TM}}$ assay (coverage $0.8 \mathrm{Mb}, 324$ genes) correlate well (19). However, below $0.5 \mathrm{Mb}$ the variance in measurements 
towards WES results increases significantly (16). Depending on the study in which a FoundationOne ${ }^{\circledR}$ assay (F1 LDT or $\mathrm{F} 1 \mathrm{CDx}^{\mathrm{TM}}$ ) is used, high TMB is defined as $\geq 9.9$ to $20 \mathrm{mut} / \mathrm{Mb}$ (7,9,21-24). A good correlation for TMB determined by WES and large panel NGS was also found for other assays. For example, another study compared WES to a 500-gene targeted NGS panel including various cancer-relevant genes. Both approaches correlated linearly (regression equation $=0.96$ ), and the NGS panel achieved a sensitivity of $>90 \%$ and a specificity of $>85 \%$ compared to WES when classifying tumors as TMB high. Cutoff for TMB high values with NGS was $15 \mathrm{mut} / \mathrm{Mb}$ in this assay (25). The Memorial Sloan Kettering-Integrated Mutation Profiling of Actionable Cancer Targets (MSK-IMPACT) is another example with a panel of 341 (version 1) to 468 genes (version 3). TMB determined by the MSK-IMPACT assays (versions $1-3$ used in this study) was highly correlated to WES (Spearman $\rho=0.86 ; \mathrm{P}<0.001$ ), patients were considered TMB high when they had $>7.4 \mathrm{mut} / \mathrm{Mb}$ (14). There are no published studies comparing the performance between each of the different large panel NGS but harmonization efforts are ongoing $(26,27)$.

As an alternative to tissue, a recent method of TMB evaluation is exploring TMB directly in circulating tumoral DNA (ctDNA) from blood/plasma (bTMB). In one study, bTMB was determined using a 394-gene panel and was compared to tissue TMB (FoundationOne ${ }^{\circledR}$ assay, not mentioned whether this was $\mathrm{F} 1 \mathrm{LDT}$ or $\mathrm{F} 1 \mathrm{CDx}^{\mathrm{TM}}$ ) and to the FoundationACT (FACT) dedicated to ctDNA assay (including only 62 genes). A total of 259 patients were evaluable for both $\mathrm{BTMB}$ and tissue TMB. Overall agreement and positive percent agreement (PPA) were $81.5 \%$ and $63.6 \%$ respectively when using the 394 -gene panel for bTMB. However, when the FACT assay was compared to tissue TMB, PPA dropped to $17 \%$, probably due to the low number of genes included in the panel. However, the performance on variant detection was similar when overlapping allele regions were compared: $93 \%$ of variants were detected in both assays (28).

\section{Causes of high TMB}

There are multiple causes for somatic mutations in cancer such as defective DNA repair, intrinsic small errors in the DNA replication machinery, enzymatic modification of DNA and exogenous exposures (29). When a cell divides, multiple errors occur, mainly at the sites of microsatellites: most of these errors are corrected during "proofreading"
(30,31). The MMR genes correct the remaining errors (32). Mutations in these damage repair genes can occur as germline mutation and as somatic mutations, both result in hypermutated tumors with a microsatellite instability-high (MSI-high) phenotype (33). Some colorectal cancers (e.g., the ones occurring in relation to the Lynch syndrome) are associated with a defect DNA MMR (34). Examples of exogenous exposures causing somatic mutations are tobacco smoking in lung cancer and ultraviolet light in skin cancers (35).

Variance of TMB number is high between and within tumor types (36-39). Tumors that are often found to have a high TMB are melanoma, NSCLC, bladder, oesophagus, colon, head and neck, gastric and endometrial cancers $(38,40,41)$. A high TMB has been associated with smoking or a molecular smoking signature in lung cancer and head and neck cancer $(38,40,41)$. Another dominant signature associated with a high TMB in lung cancer is the apolipoprotein $\mathrm{B}$ mRNA editing enzyme, catalytic polypeptide-like (APOBEC) signature $(38,40)$. Stage of disease (I/II versus III/IV) is not correlated to TMB (41).

Several other mutations such as POLD1, POLE, MSH2, POLR2A, KEAP1, PAPPA2, PXDNL, RYR1, SCN8A and SLIT3 have been associated with a high TMB, however with the exception of KEAP1 mutations they seem rare in NSCLC $(16,42,43)$.

Tobacco smoke carcinogens cause direct DNA damage and increase cancer risk by increasing the somatic mutation load as compared to non-smokers for whom the presence of a driver oncogene will be sufficient for the tumorigenesis $(44,45)$.

Most driver mutations are found in non-smoking NSCLC patients and these patients have a lower TMB. In a large dataset including 1,775 EGFR mutated, 489 ALK/ROS1 rearranged, 286 MET exon 14 mutated, 493 $\mathrm{v}$-Raf murine sarcoma viral oncogene homolog $\mathrm{B}(B R A F)$ mutated and 3,155 kirsten rat sarcoma viral oncogen (KRAS) mutated NSCLC patients, mean TMB and proportion of patients with a TMB of $>10$ or $20 \mathrm{mut} / \mathrm{Mb}$ was significantly lower for EGFR, ALK/ROS1 and MET exon 14 mutated patients compared to KRAS mutated patients. Mean TMB ranged from 3.1 to $6.2 \mathrm{mut} / \mathrm{Mb}$ for these patients compared to $10.3 \mathrm{mut} / \mathrm{Mb}$ for KRAS mutated patients. Mean TMB was similar for $B R A F$ mutated compared to KRAS mutated patients (9.7 versus $10.3 \mathrm{mut} / \mathrm{Mb}$ ), and TMB of these patient groups was comparable to all adenocarcinoma patients (mean TMB 9.1 mut/Mb). Squamous cell carcinoma patients had a slightly higher mean TMB (11.3). 
TMB was evaluated through a large NGS panel (24). Updated results (using instead of mean values the median values, median being the correct measure to use in a skewed population such as the TMB samples) including over 15,000 analyzed lung cancer cases confirmed these results, except for the lower median TMB in BRAF mutated patients (3.8 mut/Mb) (46).

A smaller series of 83 patients evaluated for TMB through the FoundationOne ${ }^{\circledR}$ assay (not mentioned whether F1 LDT or F1 $\mathrm{CDx}^{\mathrm{TM}}$ ) confirmed the lower TMB in EGFR and $A L K$ positive patients but found also a lower TMB in KRAS mutated patients when EGFR, $A L K$ and $K R A S$ mutated patients were compared to driver mutation negative patients (47). The lower TMB of EGFR mutated tumors compared to wildtype tumors was confirmed in another series of 108 resected early stage lung adenocarcinomas (48). In lung adenocarcinoma, it was found that number of mutations or neoantigens was higher in tumors with high PD-L1 and high CD8A/cytolytic activity (CYT) compared to tumors with low PD-L1 and low CD8A/CYT, the latter being an immune ignorant TME (49). In contrast to driver mutated NSCLCs with mostly a low TMB, pulmonary sarcomatoid tumors were found to have a high TMB in one retrospective series: $41 \%$ of the 122 included sarcomatoid tumors had a TMB of $>10 \mathrm{mut} / \mathrm{Mb}$ (in a large panel NGS) and $18 \%$ had a TMB of $>20 \mathrm{mut} / \mathrm{Mb}$ in one study (50). Large-cell neuroendocrine carcinoma of the lung (LCNEC) has a TMB that seems similar to that reported of adenocarcinoma patients when compared to the adenocarcinoma TMB in the series of Spigel et al. although in the latter mean was provided instead of median (median 9.9 versus mean $9.1 \mathrm{mut} / \mathrm{Mb})(24,51)$.

\section{Relevance of TMB in immunotherapy treated patients}

The goal of ICI treatment is that the patient's immune system is stimulated to reject the tumor and by doing this induces a long-lasting tumor response. There are several steps necessary to achieve this. Among them, the tumor should express neoantigens (tumour-specific, mutated peptides presented on the surface of cancer cells) that should be adequately processed and presented by the major histocompatibility complex (MHC) and afterwards these neoantigens should be recognized by T-cells as non-self to achieve an adequate anti-tumor T-cell response (52-55). The higher the number of neoantigens in a certain tumor, the higher the chance that a T-cell response is initiated to one or more of these neoantigens. It has been shown that the number of candidates neoantigens per tumor correlates well with TMB $(56,57)$.

\section{TMB and ICI outcomes in advanced NSCLC trials}

Several studies using samples of patients included in clinical trials as well as retrospective series reported ICI outcome for NSCLC patients in relation to TMB. These are described below and summarized in Table 1 .

\section{Trial data}

\section{Pembrolizumab}

One of the first series demonstrating a superior clinical efficacy of ICI in NSCLC patients with high TMB was published in 2016. TMB was evaluated with WES, 2 independent cohorts of patients treated with pembrolizumab were sequenced $(\mathrm{N}=16$ and $\mathrm{N}=18)$. A median of 200 nonsynonymous mutations was identified (range, 11-1,192). High TMB was defined as TMB above the median burden of the cohort (209 in discovery, 200 in validation cohort). In the discovery cohort $73 \%$ of patients with high TMB had durable clinical benefit compared to only $13 \%$ of those with low TMB $(\mathrm{P}=0.04)$. ORR was $63 \%$ versus $0 \%$ in those with high versus low TMB, respectively $(\mathrm{P}=0.03)$, progression free survival (PFS) was 14.5 versus 3.7 months, respectively [hazard ratio (HR) 0.19 ; $95 \%$ CI: $0.05-0.70 ; \mathrm{P}=0.01]$. Similar results were obtained in the validation cohort (56).

\section{Atezolizumab}

In a retrospective pooled analysis of the FIR and BIRCH trials (both single arm first line and beyond atezolizumab trials, including patients selected for PD-L1 positivity) a PFS benefit and a trend to an OS benefit was found in TMB high patients from the FIR and BIRCH trial. In total, 417 out of 797 patients were included in the biomarker evaluable population (BEP). TMB was determined in tissue with the F1 LDT panel. Patients with a TMB above the median $(9.9 \mathrm{mut} / \mathrm{Mb}$ ) had a PFS HR of 0.49 compared to those with a TMB below the median in first line (total of 87 patients in BEP) and a PFS HR of 0.64 in second line and beyond (total of 330 patients in BEP). HR for OS was 0.71 and 0.86 , respectively [both not significant (NS)]. With a TMB high cutoff at the $75 \%$ quantile $(16.2 \mathrm{mut} / \mathrm{Mb})$, HR for PFS in first and second line/beyond was 0.7 (NS) 


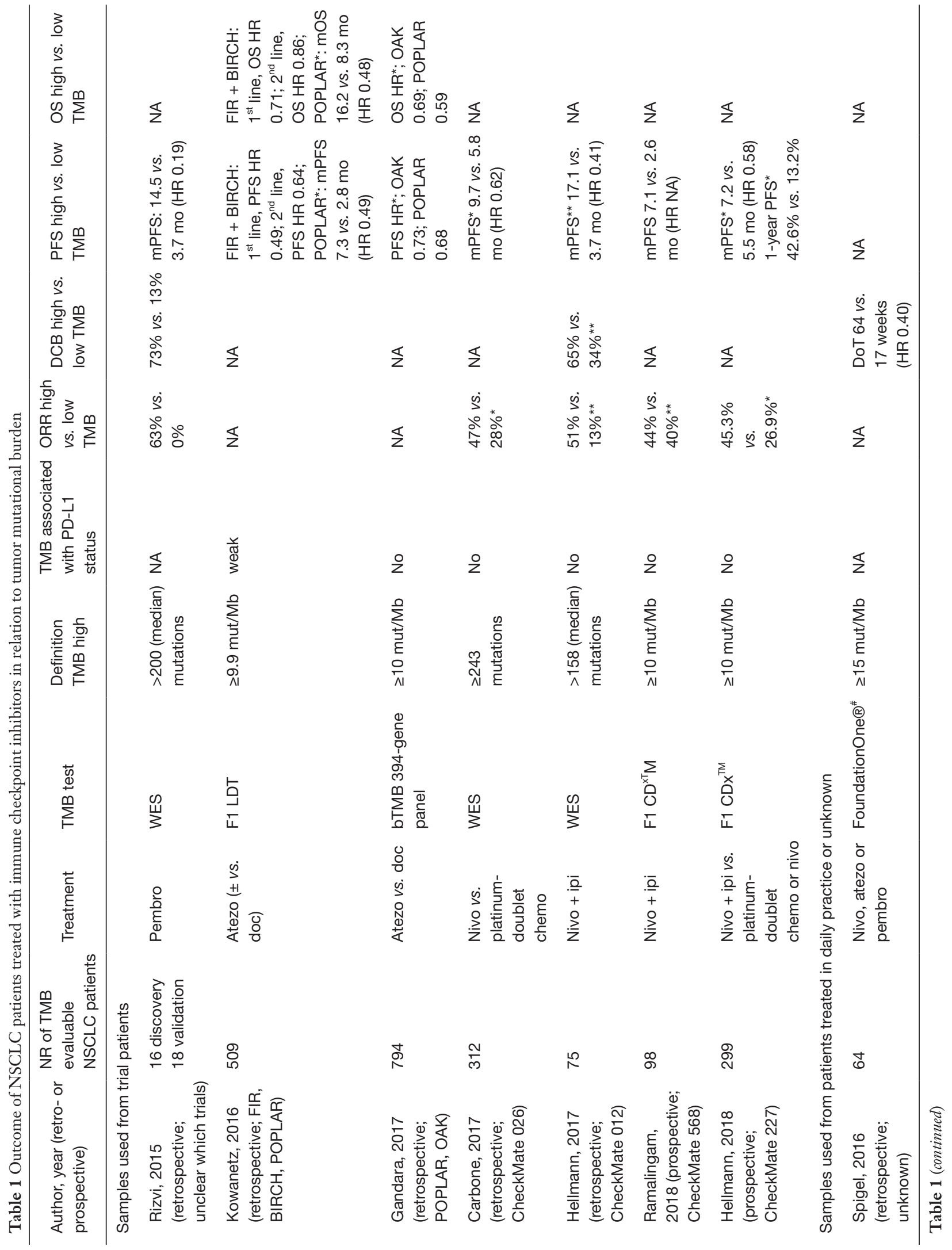




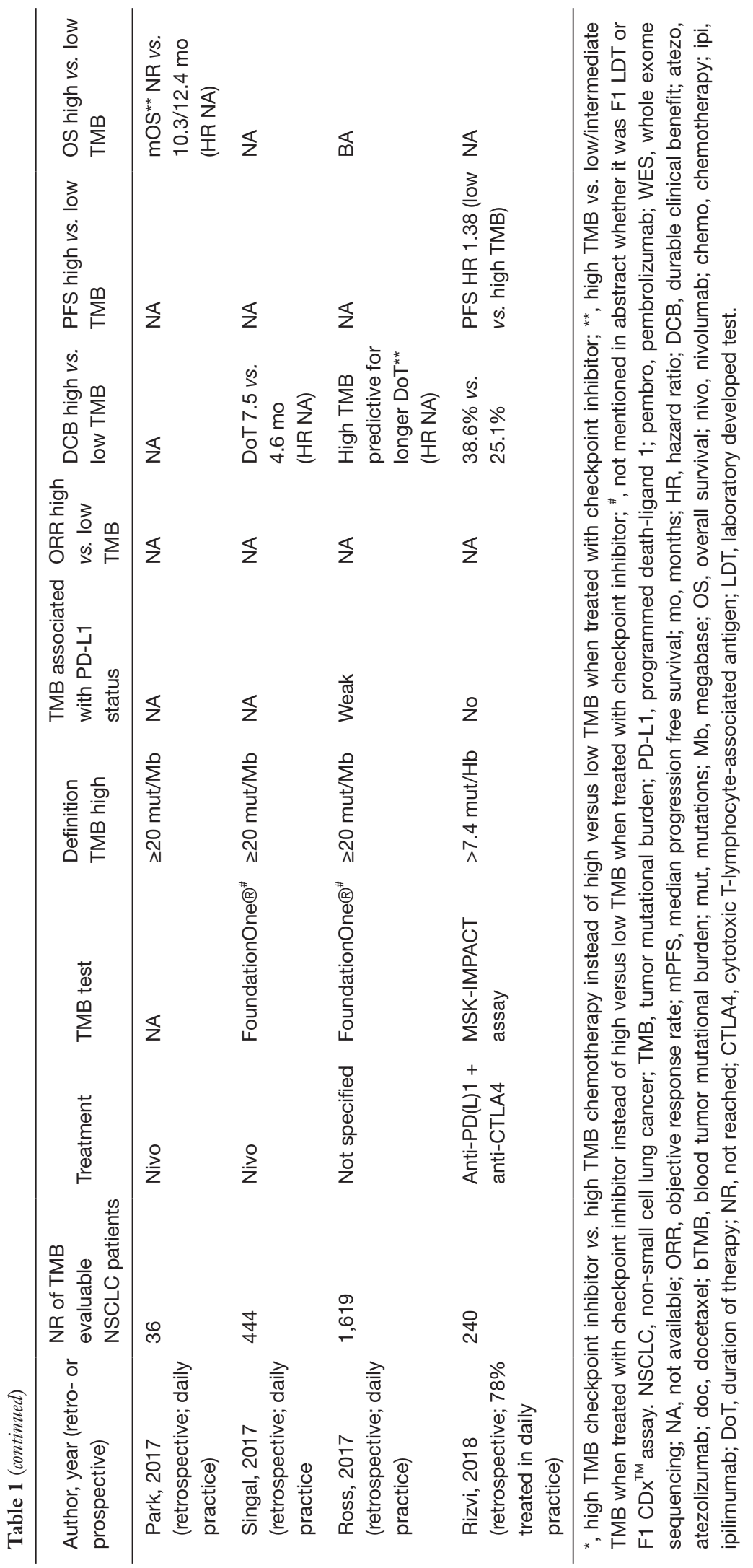


and 0.55 , respectively. HR for OS was 0.35 and 0.77 (NS) respectively. Comparable results were found for the patients in the POPLAR trial (second line and beyond atezolizumab versus docetaxel, no PD-L1 selection). In the BEP for this trial, a total of 92 out of 287 patients were included. Median PFS was 7.29 months for atezolizumab and 2.83 months for docetaxel for patients with a TMB above the median (HR 0.49; 95\% CI: 0.25-0.93). Median PFS was 8.48 and 2.83 months respectively when a TMB high cutoff at the $75 \%$ quantile was used (HR 0.49; 95\% CI: 0.19-1.3). Median OS for atezolizumab versus docetaxel was 16.23 versus 8.31 months in the $\mathrm{TMB}>$ median population (HR 0.48; 95\% CI: $0.23-1.04$ ) and not reached versus 12.16 months in the TMB $>75 \%$ quantile population (HR 0.50 ; $95 \%$ CI: $0.15-1.67)$. In the pooled analysis, TMB was weakly correlated with PD-L1 expression but not with tumor infiltrating lymphocytes (TIL). The highest ORR was found in the patient population with high TMB and PD-L1 expression (ORR just below 40\%) (21).

As is clear from the limited number of BEP patients in the atezolizumab trials, obtaining enough tissue for large panel NGS or WES is challenging. As described above, TMB can also be evaluated in blood (bTMB) and this was evaluated in an exploratory analysis of the OAK and POPLAR trials (794 out of 1,137 patients were evaluable). To determine the bTMB, 394 genes from plasma cellfree DNA were interrogated for single nucleotide variants (SNVs) and a score was based on the number of highconfidence SNVs identified. bTMB did not correlate with PD-L1, but improved PFS and OS were seen with atezolizumab compared to docetaxel for patients with a bTMB of $\geq 10$ in both the OAK and POPLAR trial [PFS HR 0.73 and 0.68, respectively; overall survival (OS) HR 0.69 and 0.59 , respectively] (58).

bTMB is currently prospectively tested in the first line single-arm phase II B-F1RST of atezolizumab monotherapy for NSCLC patients (NCT 02848651). Coprimary endpoints are ORR and PFS in the bTMB negative and positive subgroups. Arm C of the phase II/III B-FAST trial (NCT03178552) evaluates atezolizumab monotherapy versus platinum-doublet chemotherapy in advanced NSCLC patients. Primary endpoint is PFS.

\section{Nivolumab \pm ipilimumab}

The first TMB data for nivolumab came from the CheckMate 026 trial (6). In this first line randomised phase III trial, stage IV/recurring NSCLC patients with a PDL1 expression of $\geq 1 \%$ were randomised to nivolumab monotherapy or platinum-doublet chemotherapy. The trial was negative for its primary endpoint (PFS in the group of patients with a $\mathrm{PD}-\mathrm{L} 1$ expression of $\geq 5 \%$ ). However, in an exploratory analysis, TMB determined with WES correlated with outcomes. Low TMB was defined as $0-100$ mutations, median as $100-242$ mutations and high as 243 or more. A total of 312 out of 541 (58\%) randomized patients were evaluable for TMB and a slightly higher percentage of patients with a high TMB was found in the chemotherapy group compared to the nivolumab group (39\% versus $30 \%$ ). ORR as well as median PFS were higher in high TMB patients treated with nivolumab compared to high TMB patients treated with chemotherapy. ORR was $47 \%$ and $28 \%$ respectively, median PFS was 9.7 versus 5.8 months, respectively (HR 0.62; 95\% CI: 0.38-1.00). Median OS was not significantly different but cross-over rate was high $(68 \%)$. No correlation was found between TMB and PD-L1 status, but those with high TMB as well as high PD-L1 $(\geq 50 \%)$ had, when treated with nivolumab, a superior ORR (75\%) compared to those with only one of the markers defined as high (ORR 32-34\%) or when both markers were classified as low (ORR 16\%) (6).

Similar results were found in an exploratory analysis of arm G the CheckMate-012 study (nivolumab + ipilimumab). Totally, 75 of the 197 included patients had sufficient tumor tissue and paired blood for WES. ORR, durable clinical benefit (DCB) and PFS were significantly higher in those with a TMB above the median [158] compared to those with a TMB below the median. ORR was $51 \%$ versus $13 \%$ $(\mathrm{P}=0.0005)$, $\mathrm{DCB}$ was $65 \%$ versus $34 \%(\mathrm{P}=0.011)$, median PFS was 17.1 months for those with a high TMB compared to 3.7 months for those with a low TMB (HR 0.41; 95\% CI: $0.23-0.73 ; \mathrm{P}=0.0024)$. $\mathrm{PD}-\mathrm{L} 1$ expression was not correlated to TMB ( $\mathrm{P}=0.804)$. Patients with high TMB as well as $\mathrm{PD}$ L1 expression $\geq 1 \%$ had the best ORR and PFS (57).

In the CheckMate 568 trial, a single-arm phase II study of first line nivolumab plus ipilimumab in advanced NSCLC, the investigators sought to identify an appropriate TMB cut-off to select patients for this first line ICI combination. Primary endpoint was ORR, one of the secondary endpoints was ORR by TMB. In contrast to the Checkmate 026 trial, TMB was determined through large panel NGS with the validated $\mathrm{F} 1 \mathrm{CDx}^{\mathrm{TM}}$ assay. 288 patients were included, and overall ORR was $27 \% .98$ patients (34\%) were evaluable for TMB. ORR plateaud with the threshold of $\geq 10$ mutations/Mb. ORR was $4 \%$ with $\mathrm{TMB}<5,10 \%$ with $\mathrm{TMB}<10,44 \%$ with $\mathrm{TMB} \geq 10$ and $39 \%$ with TMB $\geq 15 \mathrm{mut} / \mathrm{Mb}$. With receiver operating characteristic (ROC) 
curves optimal classification performance was found at 10 mut/Mb. Median PFS (95\% CI) was higher in TMB high patients [7.1 (3.6-11.3) versus 2.6 (1.5-5.4) months] (9).

The first randomized phase III trial evaluating ICI outcome in NSCLC patients predefined in relation to TMB was the CheckMate 227 trial (7). In this open label trial two separate cohorts of NSCLC patients [one cohort with PD-L1 expression of $\geq 1 \%(\mathrm{~N}=1,189)$, the other cohort with PD-L1 expression of $<1 \%(\mathrm{~N}=550)$ ] were randomized to nivolumab plus ipilimumab, platinumdoublet chemotherapy or nivolumab monotherapy (when PD-L1 < $\%$ nivolumab combined with platinum-based chemotherapy). The initial primary endpoint was OS with nivolumab or nivolumab plus ipilimumab versus chemotherapy on the basis of PD-L1 expression $(\geq 1 \%$ versus $<1 \%$ ). When the results of the CheckMate 568 trial became available, the trial was amended to add a co-primary endpoint of PFS with nivolumab plus ipilimumab versus chemotherapy in a patient population on the basis of TMB (i.e., patients were not tested for TMB at baseline and patients were not stratified for TMB). Cutoff for high TMB was 10 mut/Mb based on the results of the CheckMate 568 trial (TMB again determined by the $\mathrm{F} 1 \mathrm{CDx}^{\mathrm{TM}}$ assay) (56). One of the secondary endpoints was PFS with nivolumab versus chemotherapy among patients with a TMB of $\geq 13 \mathrm{mut} / \mathrm{Mb}$ and a PD-L1 level of $\geq 1 \%$. A total of 1,004 of $1,739(57.7 \%)$ included patients (across all treatment groups) were evaluable for TMB. In total, 330 of 583 $(56.6 \%)$ nivolumab + ipilimumab treated patients and 349 of $583(59.9 \%)$ chemotherapy treated patients were evaluable for TMB. A total of 139 of 330 (41.1\%) and 160 of $349(45.8 \%)$ patients were TMB high in the nivolumab + ipilimumab and chemotherapy arms, respectively. Baseline characteristics (including PD-L1) were well balanced between these TMB high groups. Median PFS was significantly higher for patients with a high TMB treated with nivolumab plus ipilimumab compared to these treated with chemotherapy and was 7.2 (95\% CI: 5.5-13.2) versus 5.5 (95\% CI: 4.4-5.8) months (HR 0.58; 95\% CI: 0.41-0.81; $\mathrm{P}<0.001)$. One-year PFS rate was $42.6 \%$ versus $13.2 \%$ respectively, and ORR was $45.3 \%$ versus $26.9 \%$ respectively. After adjusting for baseline PD-L1, sex, tumor type and performance status a comparable $\mathrm{HR}$ for PFS was found (HR 0.57; 95\% CI: 0.40-0.80; $\mathrm{P}<0.001$ ). In contrast, for the subgroup of patients with a low TMB, no PFS benefit was found for nivolumab plus ipilimumab versus chemotherapy. Median PFS was not significantly different and was 3.2 (95\% CI: 2.7-4.3) and 5.5 (95\% CI: 4.3-5.6) months, respectively (HR 1.07; 95\% CI: 0.84-1.35). Interestingly, and in contrast to the CheckMate 026 trial, PFS was not superior for nivolumab versus chemotherapy in the TMB $\geq 13 \mathrm{mut} / \mathrm{Mb}$ and PD-L1 level of $\geq 1 \%$ (HR 0.95; 95\% CI: 0.61-1.48; $\mathrm{P}=0.78)(7)$.

\section{Retrospective data from daily practice}

\section{Anti-PD(L)1 therapy}

In an analysis of 64 NSCLC patients treated with nivolumab, pembrolizumab or atezolizumab, a high TMB (FoundationOne® assay (not mentioned whether F1 LDT or F1 $\mathrm{CDx}^{\mathrm{TM}}$ ), TMB high: $\geq 15$ mut/Mb) was associated with a longer duration of therapy: median was 64 versus 17 weeks (HR 0.396; 95\% CI: 0.190-0.825; P=0.010). Moreover MSI-high status correlated strongly with TMB (24).

\section{Nivolumab}

A numerically higher median OS for high TMB NSCLC patients treated with nivolumab was found in a small study $(\mathrm{N}=36)$ : median OS (95\% CI) was not reached for TMB high ( $\geq 20 \mathrm{mut} / \mathrm{Mb}), 10.3$ (4.8-15.7) for intermediate TMB (6-19 mut/Mb) and 12.4 (7.1-17.1) months $(\mathrm{P}=0.211)$. Interestingly, a clinical score (iSEND) based on gender, performance status, neutrophil-to-lymphocyte ratio (NLR) and deltaNLR correlated significantly with OS. Median OS (95\% CI) was 15.7 (10.8-20.6), 10.3 (4.8-15.7) and 3.7 (0-7.8) months for iSEND good, intermediate and poor groups, respectively $(\mathrm{P}<0.001)$. TMB and iSEND were not combined to one predictive score (59).

In a series of 444 NSCLC patients treated with nivolumab, high TMB (determined through the FoundationOne ${ }^{\circledR}$ (not mentioned whether F1 LDT or F1 $\mathrm{CDx}^{\mathrm{TM}}$ ) assay, TMB high defined as $>20$ mut/Mb), patients with a high TMB had a longer duration of nivolumab therapy ( 7.5 versus 4.6 months, $\mathrm{P}=0.001)$ as well as a longer median OS (not reached versus 10 months, $\mathrm{P}<0.01$ ). In this series, PD-L1 status (available for 282 patients) was not associated with outcome (23).

In another large series (1,619 samples from the FoundationCORE database and 2,139 samples from the Flatiron Health Analytic Database) of NSCLC patients treated with IC with available TMB [FoundationOne ${ }^{\circledR}$ assay (not mentioned whether F1 LDT or F1 CDx ${ }^{\mathrm{TM}}$ )] and PD-L1 status, TMB and PD-L1 correlated weakly. Median TMB was 8.1 mut/Mb and $14.5 \%$ had a high TMB (defined as $\geq 20$ mut/Mb). PD-L1 status was not associated 
with duration of therapy but high TBM predicted for longer duration of therapy $(\mathrm{P}=0.001)$. Interestingly, STK11 mutations correlated with a high TMB/low-PD-L1 status and in a preliminary analysis an inferior outcome on ICI. $B R A F$ mutations were correlated with a longer duration of therapy irrespective of TMB (22).

The use of TMB determined by a large NGS panel (MSK-IMPACT assay) has also been evaluated in 240 patients treated with anti-PD-(L)1 or anti-PD-(L)1 plus anti-CTLA4 combination therapy. 186 (78\%) of the treated patients were treated outside of a clinical trial. Totally, 29\% of the patients had DCB and TMB was significantly higher in those with DCB compared to those without (median 8.5 versus $6.6 \mathrm{SNVs} / \mathrm{Mb}, \mathrm{P}=0.0062)$. $\mathrm{DCB}$ was $38.6 \%$ vs. $25.1 \%$ in those with a TMB above versus below the 50 th percentile $(\mathrm{P}<0.001)$. PFS HR was $1.38(\mathrm{P}=0.024)$. TMB was also evaluated in a non-ICI, chemotherapy treated cohort of NSCLC patients: an inverse relationship between TMB and survival was identified (14). PD-L1 expression level was also significantly correlated with PFS (HR 0.526; $\mathrm{P}=0.011$ for $\mathrm{PD}-\mathrm{L} 1 \geq 1 \%$ versus $0 \%$ ) but not with $\mathrm{TMB}$. Patients with high TMB and PD-L1 positivity $(\geq 1 \%)$ had the highest rate of DCB (50\%) (14).

\section{Drawbacks of TMB}

Determining TMB becomes increasingly feasible with the availability of NGS technology. However, the determination of TMB on tissue raises several limitations in term of sample consumption and timing of the results. For example, for the commercially available $\mathrm{F} 1 \mathrm{CDx}^{\mathrm{TM}}$ test, a tumor block or 10 unstained tumor slides cut at 4-5 microns thickness are preferred with an optimum of $25 \mathrm{~mm}^{2}$ surface area and at least $20 \%$ (preferred $30 \%$ ) of tumor nuclei. In 2018, turnaround time is approximately two weeks, and cost is between 2,500 and 5,000 US dollars although it is expected that price will decrease with time $(18,19,60)$.

Secondly, the attrition rate due to sample quality and quantity could be higher than current molecular testing. The percentage of TMB evaluable patients enrolled in clinical trials is lower than that for PD-L1. For example, in the CheckMate 568 trial, $88 \%$ of patients were evaluable for PD-L1 but only $34 \%$ were evaluable for TMB $\left(\mathrm{F} 1 \mathrm{CDx}^{\mathrm{TM}}\right)$ (9).

Furthermore, TMB testing is not standardized for the different assays used and the definition of high TMB varies across studies from $\geq 7.4$ to $20 \mathrm{mut} / \mathrm{Mb}$ when measured with a large NGS panel $(7,9,14,21-24,58,59)$. As already mentioned, harmonization efforts are ongoing (26,27), but our opinion is that more prospective clinical trials will be needed to correctly determine the threshold and validate the clinical utilization of the TMB result.

Besides quantity and quality of the tissue, time, costs and defining the optimal cutoff there are drawbacks of measuring TMB as not only total TMB influences outcome on ICI. For example, when measuring TMB from a single sample, both clonal and subclonal neoantigens are counted in the TMB. Clonal neoantigens are present in all tumor cells while subclonal neoantigens are only present in a subset of tumor cells. In a series of 31 tumors from NSCLC patients treated with pembrolizumab it was shown that especially those with a high TMB as well as a low neoantigen subclonal fraction (defined as $<5 \%$ subclonal) had the most durable clinical benefit with pembrolizumab. The authors also demonstrated that T-cells only recognized clonal neoantigens and not subclonal neoantigens. This is important as chemotherapy seems to enhance TMB mainly through the increase of subclonal neoantigens. Even when T-cells are generated against these subclonal antigens, these T-cells will not be able to control all tumor cells (61). Similar results were found in an exploratory analysis of the CheckMate 012 trial: clonal predicted neoantigen burden was more predictive of improved PFS compared with total predicted neoantigen burden (57).

Not only the load of neoantigens but also the neoantigen fitness should also be taken into account (62), This can be modelled by not only using information on the frequency of the clone but also using amplitude of MHC presentation (using relative MHC affinity of wild-type and mutant peptide) and $\mathrm{T}$-cell recognition probability (62).

\section{Other factors influencing immunotherapy outcome}

Besides PD-L1 status and TMB, outcome on ICI can be influenced by several factors. An exhaustive coverage of biomarkers and other factors that influence outcome on ICI is outside of the scope of this review. Some examples are summarized here. It has been demonstrated that some cancer types have a better outcome with ICI treatment than would be expected from the TMB and for other tumors outcome is worse (17). Other factors such as viral antigen presentation on tumors (e.g., Merkel-cell carcinoma) may also result in a favourable ORR despite a lower TMB (63). Several oncogenic pathways can also have an impact on evasion of antitumor response, as was reviewed in Spranger 
et al. (64). In summary, defective critical steps in antitumour T-cell priming might result in a poor outcome despite high TMB. For example, functional dendritic cells are necessary for cross-presenting tumor neoantigens through the MHC class I pathway. Moreover, there are a certain number of gain-of-function alterations such as WNT-betacatenin signalling or gain of MYC function that result in reduced T-cell recruitment and reduced T-cell activation and infiltration, respectively. Loss-of-function alterations such as LKB1 (STK11) mutations, PTEN loss, and TP53 loss can result in decreased T-cell infiltration, reduced T-cell priming and decreased $\mathrm{T}$-cell infiltration, respectively. Other aberrations in oncogenic pathways, such as mutations in isocitrate dehydrogenase 1 and 2 (IDH1 and IDH2) are associated with reduced T-cell infiltration while STAT3 signalling can decrease expression of pro-inflammatory cytokines $(57,64)$. It has been shown that TILs represent both a predictive and a prognostic biomarker $(65,66)$.

Furthermore, gene expression profiling performed in longitudinal tumor biopsies showed dynamic changes in multiple genes after the initiation of PD-1 therapy. These immune signatures could also be a complementary solution to guide treatment decisions. Targeted gene expression panels quantify the specific RNA expression profiles from formalin-fixed paraffin embedded (FFPE) biopsy. The quantification of the RNA expression will comprehensively delineate an inflamed tumor microenvironment. Immune expression profiling has the potential to accurately determine the inflammatory status of a tumor better than the PD-L1 status alone. The correlations between RNAseq and those signatures have been explored and seem very acceptable (67). Several approaches are proposed with RNA extracted from FFPE: a 25-gene interferon-gamma (IFN- $\gamma$ ) signature derived from crude extract (EdgeSeq, HTG Molecular Diagnostics Tucson, AZ, USA) and 700 genes from isolated RNA (NanoString Technologies, Seattle, WA, USA). For lung cancer, several trials have used this option: for example, the POPLAR trial with atezolumab and the T-effector/IFN- $\gamma$ signature. An OS HR of 0.43 (95\% CI: 0.24-0.77) was found for atezolizumab versus docetaxel in the high gene expression level group compared to an OS HR of 1.1 (95\% CI: 0.68-1.76) for atezolizumab versus docetaxel in the low gene expression group (68).

It is also important to take into account exogenous factors such as use of antibiotics and steroids. It has been shown in a retrospective study $(\mathrm{N}=360$, of which 239 NSCLC) that use of antibiotics within 30 days of ICI start is associated with a worse outcome compared to patients that did not use antibiotics. For NSCLC patients, median PFS was 1.9 versus 3.8 months, respectively (HR 1.5 ; $95 \% \mathrm{CI}$ : $1.0-2.2 ; \mathrm{P}=0.03)$. Median $\mathrm{OS}$ was 7.9 versus 24.6 months, respectively (HR 4.4; 95\% CI: $2.6-7.7 ; \mathrm{P}<0.01$ ). Use of antibiotics retained its significance for $\mathrm{OS}$ in multivariate analysis (69). An imbalance in gut microbiota has also been associated with poor outcomes on ICI, and it is suggested that this might be caused by use of antibiotics (70).

Baseline steroid use (prednisolon equivalent of $\geq 10 \mathrm{mg} /$ day) was also associated with worse outcome on ICI in a retrospective series of 640 NSCLC patients and steroid use retained its significance in multivariate analysis [HR for PFS $1.31(\mathrm{P}=0.03)$, HR for OS $1.66(\mathrm{P}<0.001)](71)$.

\section{Future: TMB and other biomarkers to guide treatment decisions}

There are besides PD-L1 and TMB multiple parameters associated with ICI outcome (e.g., prognostic scores such as LIPI and dNLR, TILs, T-effector/IFN- $\gamma$ signature, general immune fitness, soluble inhibitors, tumor metabolism, microbiome), and as such it would be interesting to combine several parameters $(16,52,66,68,72)$.

Challenge when combining multiple biomarkers is the increasing number of subgroups that is created. For example, with two biomarkers there are already four subgroups and this doubles to eight when there are three biomarkers combined. It then becomes increasingly difficult to define cutoffs and interdependence. In reports of clinical trials, only PD-L1 and TMB were combined.

However, obtaining enough tissue to perform all analysis necessary to make a treatment decision (define histological subtype, test for molecular drivers, PD-L1 and TMB) can be difficult. Furthermore, most predictive biomarkers are expensive to perform in daily routine. Exceptions are the predictive scores using laboratory values already determined in routine clinical care as the LIPI score which uses only pretreatment neutrophils, lymphocytes and LDH (72). Now, the performance of all those scores should be evaluated and compared with PD-L1 or TMB testing.

\section{Conclusions}

Anti-PD(L)1 therapy has become standard of care for NSCLC, and some of the combination strategies with chemotherapy (or anti-CTLA4 therapy) are likely to become standard of care in the near future for certain subgroups of patients. With all of these possible treatment options, 
biomarkers are needed to identify which subgroup of patients is likely to benefit the most from a certain therapy. Although not perfect, a high PD-L1 expression level can aid in selecting the right patient population for monotherapy pembrolizumab. TMB is an interesting biomarker, however assays and definition for high TMB should be standardized and it is challenging to obtain an adequate amount of tissue. Importantly, high TMB predicted for improved PFS with nivolumab plus ipilimumab but OS data of the CheckMate 227 are still immature and data for nivolumab monotherapy versus chemotherapy are conflicting. When assays are standardized and an OS benefit is shown, TMB in combination with PD-L1 expression level could help in defining subgroups of patients that will likely benefit from monotherapy or combination strategies.

\section{Acknowledgements}

L Hendriks was the recipient of a DUERTECC/EURONCO grant for the year 2017-2018.

\section{Footnote}

Conflicts of Interest: LE Hendriks: institutional research funding Roche, advisory board: Boehringer, BMS, travel reimbursement: Roche, BMS. E Rouleau: Advisory board: AstraZeneca, Roche, BMS - Travel reimbursement: BMS, AstraZeneca. B Besse: Institutional grants for clinical and translational research AstraZeneca, BMS, BoehringerIngelheim, Lilly, Pfizer, Roche-Genentech, Sanofi-Aventis, Servier, Onxeo, OncoMed, Inivata, OSE Pharma, Loxo.

\section{References}

1. Reck M, Rodriguez-Abreu D, Robinson AG, et al. Pembrolizumab versus Chemotherapy for PD-L1Positive Non-Small-Cell Lung Cancer. N Engl J Med 2016;375:1823-33.

2. Herbst RS, Baas P, Kim DW, et al. Pembrolizumab versus docetaxel for previously treated, PD-L1-positive, advanced non-small-cell lung cancer (KEYNOTE-010): a randomised controlled trial. Lancet 2016;387:1540-50.

3. Borghaei H, Paz-Ares L, Horn L, et al. Nivolumab versus Docetaxel in Advanced Nonsquamous Non-Small-Cell Lung Cancer. N Engl J Med 2015;373:1627-39.

4. Brahmer J, Reckamp KL, Baas P, et al. Nivolumab versus Docetaxel in Advanced Squamous-Cell Non-Small-Cell Lung Cancer. N Engl J Med 2015;373:123-35.
5. Rittmeyer A, Barlesi F, Waterkamp D, et al. Atezolizumab versus docetaxel in patients with previously treated non-small-cell lung cancer (OAK): a phase 3, openlabel, multicentre randomised controlled trial. Lancet 2017;389:255-65.

6. Carbone DP, Reck M, Paz-Ares L, et al. First-Line Nivolumab in Stage IV or Recurrent Non-Small-Cell Lung Cancer. N Engl J Med 2017;376:2415-26.

7. Hellmann MD, Ciuleanu TE, Pluzanski A, et al. Nivolumab plus Ipilimumab in Lung Cancer with a High Tumor Mutational Burden. N Engl J Med 2018;378:2093-104.

8. Antonia S, Goldberg SB, Balmanoukian A, et al. Safety and antitumour activity of durvalumab plus tremelimumab in non-small cell lung cancer: a multicentre, phase $1 \mathrm{~b}$ study. Lancet Oncol 2016;17:299-308.

9. Ramalingam S, Hellmann M, Awad MM, et al. CT078 - Tumor mutational burden (TMB) as a biomarker for clinical benefit from dual immune checkpoint blockade with nivolumab (nivo) + ipilimumab (ipi) in first-line (1L) non-small cell lung cancer (NSCLC): identification of TMB cutoff from CheckMate 568. Cancer Res 2018;78.

10. Gandhi L, Rodriguez-Abreu D, Gadgeel S, et al. Pembrolizumab plus Chemotherapy in Metastatic Non-Small-Cell Lung Cancer. N Engl J Med 2018;378:2078-92.

11. Reck M, Socinski MA, Cappuzzo F, et al. LBA1_PR Primary PFS and safety analyses of a randomised Phase III study of carboplatin + paclitaxel +/- bevacizumab, with or without atezolizumab in $1 \mathrm{~L}$ non-squamous metastatic NSCLC (IMpower150). Ann Oncol 2017;28:xi31.

12. Le DT, Durham JN, Smith KN, et al. Mismatch repair deficiency predicts response of solid tumors to PD-1 blockade. Science 2017;357:409-13.

13. Buttner R, Gosney JR, Skov BG, et al. Programmed Death-Ligand 1 Immunohistochemistry Testing: A Review of Analytical Assays and Clinical Implementation in NonSmall-Cell Lung Cancer. J Clin Oncol 2017;35:3867-76.

14. Rizvi H, Sanchez-Vega F, La K, et al. Molecular Determinants of Response to Anti-Programmed Cell Death (PD)-1 and Anti-Programmed Death-Ligand 1 (PD-L1) Blockade in Patients With Non-Small-Cell Lung Cancer Profiled With Targeted Next-Generation Sequencing. J Clin Oncol 2018;36:633-41.

15. Johnson DB, Frampton GM, Rioth MJ, et al. Targeted Next Generation Sequencing Identifies Markers of Response to PD-1 Blockade. Cancer Immunol Res 2016;4:959-67. 
16. Chalmers ZR, Connelly CF, Fabrizio D, et al. Analysis of 100,000 human cancer genomes reveals the landscape of tumor mutational burden. Genome Med 2017;9:34.

17. Yarchoan M, Hopkins A, Jaffee EM. Tumor Mutational Burden and Response Rate to PD-1 Inhibition. N Engl J Med 2017;377:2500-1.

18. Frampton GM, Fichtenholtz A, Otto GA, et al. Development and validation of a clinical cancer genomic profiling test based on massively parallel DNA sequencing. Nat Biotechnol 2013;31:1023-31.

19. FoundationOne CDx. Foundation One - order Foundation One technical information. 2018. Available online: https://www.foundationmedicine.com/genomic-testing/ foundation-one-cdx\#ordering

20. Szustakowski J, Green G, Geese W, et al. Evaluation of tumor mutation burden as a biomarker for immune checkpoint inhibitor efficacy: A calibration study of whole exome sequencing with FoundationOne ${ }^{\circledR}$. Cancer Res $2018 ; 78$.

21. Kowanetz M, Zou W, Shames DS, et al. Tumor mutation burden (TMB) is associated with improved efficacy of atezolizumab in $1 \mathrm{~L}+$ and $2 \mathrm{~L}+$ NSCLC patients. J Thorac Oncol 2017;12:S321-2.

22. Ross J, Goldberg M, Albacker L, et al. Immune Checkpoint Inhibitor (ICPI) Efficacy and Resistance Detected by Comprehensive Genomic Profiling (CGP) in Non-Small Cell Lung Cancer (NSCLC). Ann Oncol 2017;28:v403-27.

23. Singal G, Miller P, Agarwala V, et al. Analyzing biomarkers of cancer immunotherapy (CIT) response using a real-world clinico-genomic database. Ann Oncol 2017;28:v403-27.

24. Spigel D, Schrock A, Fabrizio D, et al. Total mutation burden (TMB) in lung cancer (LC) and relationship with response to $\mathrm{PD}-1 / \mathrm{PD}-\mathrm{L} 1$ targeted therapies. J Clin Oncol 2016;34:9017.

25. So A, Kaplan S, Zhao Z, et al. Accurate measurement of tumor mutation burden through tumor-only sequencing using a 500-gene panel. Cancer Res 2018;78.

26. Karow J. Two Initiatives Seek to Harmonize Tumor Mutational Burden Testing. 2018. Available online: https://www.genomeweb.com/cancer/two-initiativesseek-harmonize-tumor-mutational-burden-testing\#. WzHi_Xrj-Fw

27. Research FoC. TMB Harmonization Working Group Meeting. 2018. Available online: https://www.focr.org/ events/tmb-harmonization-working-group-meeting

28. Fabrizio D, Lieber DS, Malboeuf C, et al. A blood-based next-generation sequencing assay to determine tumor mutational burden (bTMB) is associated with benefit to an anti-PD-L1 inhibitor, atezolizumab. Cancer Res 2018;78.

29. Stratton MR, Campbell PJ, Futreal PA. The cancer genome. Nature 2009;458:719-24.

30. Bebenek K, Kunkel TA. Functions of DNA polymerases. Adv Protein Chem 2004;69:137-65.

31. Kunkel TA, Erie DA. DNA mismatch repair. Annu Rev Biochem 2005;74:681-710.

32. Arana ME, Kunkel TA. Mutator phenotypes due to DNA replication infidelity. Semin Cancer Biol 2010;20:304-11.

33. Peltomaki P. Role of DNA mismatch repair defects in the pathogenesis of human cancer. J Clin Oncol 2003;21:1174-9.

34. Pena-Diaz J, Bregenhorn S, Ghodgaonkar M, et al. Noncanonical mismatch repair as a source of genomic instability in human cells. Mol Cell 2012;47:669-80.

35. Pfeifer GP. Environmental exposures and mutational patterns of cancer genomes. Genome Med 2010;2:54.

36. Schreiber RD, Old LJ, Smyth MJ. Cancer immunoediting: integrating immunity's roles in cancer suppression and promotion. Science 2011;331:1565-70.

37. Lawrence MS, Stojanov P, Polak P, et al. Mutational heterogeneity in cancer and the search for new cancerassociated genes. Nature 2013;499:214-8.

38. Alexandrov LB, Nik-Zainal S, Wedge DC, et al. Signatures of mutational processes in human cancer. Nature 2013;500:415-21.

39. Vogelstein B, Papadopoulos N, Velculescu VE, et al. Cancer genome landscapes. Science 2013;339:1546-58.

40. Zehir A, Benayed R, Shah RH, et al. Mutational landscape of metastatic cancer revealed from prospective clinical sequencing of 10,000 patients. Nat Med 2017;23:703-13.

41. Colli LM, Machiela MJ, Myers TA, et al. Burden of Nonsynonymous Mutations among TCGA Cancers and Candidate Immune Checkpoint Inhibitor Responses. Cancer Res 2016;76:3767-72.

42. Liu L, Ruiz J, O'Neill SS, et al. Favorable outcome of patients with lung adenocarcinoma harboring POLE mutations and expressing high PD-L1. Mol Cancer 2018;17:81.

43. Frank R, Scheffler M, Merkelbach-Bruse S, et al. Clinical and pathological characteristics of KEAP1- and NFE2L2mutated non-small cell lung carcinoma (NSCLC). Clin Cancer Res 2018;24:3087-96.

44. Pfeifer GP, Denissenko MF, Olivier M, et al. Tobacco smoke carcinogens, DNA damage and p53 mutations in 
smoking-associated cancers. Oncogene 2002;21:7435-51.

45. Alexandrov LB, Ju YS, Haase K, et al. Mutational signatures associated with tobacco smoking in human cancer. Science 2016;354:618-22.

46. Schrock A, Sharma N, Peled N, et al. Updated Dataset Assessing Tumor Mutation Burden (TMB) as a Biomarker for Response to PD-1/PD-L1 Targeted Therapies in Lung Cancer (LC). J Thorac Oncol 2017;12:S422.

47. Davis A, Chae Y, Agte S, et al. Association of tumor mutational burden with smoking and mutation status in non-small cell lung cancer (NSCLC). J Clin Oncol 2017;35:abstract 24.

48. Kadara H, Choi M, Zhang J, et al. Whole-exome sequencing and immune profiling of early-stage lung adenocarcinoma with fully annotated clinical follow-up. Ann Oncol 2017;28:75-82.

49. Chen YP, Zhang Y, Lv JW, et al. Genomic Analysis of Tumor Microenvironment Immune Types across 14 Solid Cancer Types: Immunotherapeutic Implications. Theranostics 2017;7:3585-94.

50. Schrock AB, Li SD, Frampton GM, et al. Pulmonary Sarcomatoid Carcinomas Commonly Harbor Either Potentially Targetable Genomic Alterations or High Tumor Mutational Burden as Observed by Comprehensive Genomic Profiling. J Thorac Oncol 2017;12:932-42.

51. Chae Y, Tamragouri K, Chung J, et al. Genomic alterations (GA) and tumor mutational burden (TMB) in large cell neuroendocrine carcinoma of lung (L-LCNEC) as compared to small cell lung carcinoma (SCLC) as assessed via comprehensive genomic profiling (CGP). J Clin Oncol 2017;35.

52. Chen DS, Mellman I. Elements of cancer immunity and the cancer-immune set point. Nature 2017;541:321-30.

53. Chen DS, Mellman I. Oncology meets immunology: the cancer-immunity cycle. Immunity 2013;39:1-10.

54. Schumacher TN, Schreiber RD. Neoantigens in cancer immunotherapy. Science 2015;348:69-74.

55. Gubin MM, Artyomov MN, Mardis ER, et al. Tumor neoantigens: building a framework for personalized cancer immunotherapy. J Clin Invest 2015;125:3413-21.

56. Rizvi NA, Hellmann MD, Snyder A, et al. Cancer immunology. Mutational landscape determines sensitivity to PD-1 blockade in non-small cell lung cancer. Science 2015;348:124-8.

57. Hellmann MD, Nathanson T, Rizvi H, et al. Genomic Features of Response to Combination Immunotherapy in Patients with Advanced Non-Small-Cell Lung Cancer. Cancer Cell 2018;33:843-52.e4.
58. Gandara D, Kowanetz M, Mok T, et al. Blood-based biomarkers for cancer immunotherapy: Tumor mutational burden in blood (bTMB) is associated with improved atezolizumab (atezo) efficacy in 2L+ NSCLC (POPLAR and OAK). Ann Oncol 2017;28.

59. Park W, Lopes G, Kwon D, et al. Correlating ISEND and Tumor Mutation Burden (TMB) with Clinical Outcomes of Advanced Non-Small Cell Lung Cancer (ANSCLC) Patients on Nivolumab. J Thorac Oncol 2017;12:S2005-6.

60. Nanos J. Google to offer FoundationOne cancer tests to employees. 2014. Available online: http://www.betaboston. com/news/2014/11/05/google-to-offer-foundationonecancer-tests-to-employees/

61. McGranahan N, Furness AJ, Rosenthal R, et al. Clonal neoantigens elicit $\mathrm{T}$ cell immunoreactivity and sensitivity to immune checkpoint blockade. Science 2016;351:1463-9.

62. Luksza M, Riaz N, Makarov V, et al. A neoantigen fitness model predicts tumour response to checkpoint blockade immunotherapy. Nature 2017;551:517-20.

63. Nghiem PT, Bhatia S, Lipson EJ, et al. PD-1 Blockade with Pembrolizumab in Advanced Merkel-Cell Carcinoma. N Engl J Med 2016;374:2542-52.

64. Spranger S, Gajewski TF. Impact of oncogenic pathways on evasion of antitumour immune responses. Nat Rev Cancer 2018;18:139-47.

65. Donnem T, Hald SM, Paulsen EE, et al. Stromal CD8+ T-cell Density-A Promising Supplement to TNM Staging in Non-Small Cell Lung Cancer. Clin Cancer Res 2015;21:2635-43.

66. Tumeh PC, Harview CL, Yearley JH, et al. PD-1 blockade induces responses by inhibiting adaptive immune resistance. Nature 2014;515:568-71.

67. Prat A, Navarro A, Pare L, et al. Immune-Related Gene Expression Profiling After PD-1 Blockade in Non-Small Cell Lung Carcinoma, Head and Neck Squamous Cell Carcinoma, and Melanoma. Cancer Res 2017;77:3540-50.

68. Fehrenbacher L, Spira A, Ballinger M, et al. Atezolizumab versus docetaxel for patients with previously treated non-small-cell lung cancer (POPLAR): a multicentre, open-label, phase 2 randomised controlled trial. Lancet 2016;387:1837-46.

69. Derosa L, Hellmann MD, Spaziano M, et al. Negative association of antibiotics on clinical activity of immune checkpoint inhibitors in patients with advanced renal cell and non-small cell lung cancer. Ann Oncol 2018;29:1437-44.

70. Routy B, Le Chatelier E, Derosa L, et al. Gut microbiome influences efficacy of PD-1-based immunotherapy against 
epithelial tumors. Science 2018;359:91-7.

71. Arbour K, Mezquita L, Long N, et al., editors. Deleterious effect of baseline steroids on efficacy of PD-(L)1 blockade in patients with NSCLC. J Clin Oncol 2018;36.

72. Mezquita L, Auclin E, Ferrara R, et al. Association of

Cite this article as: Hendriks LE, Rouleau E, Besse B. Clinical utility of tumor mutational burden in patients with non-small cell lung cancer treated with immunotherapy. Transl Lung Cancer Res 2018;7(6):647-660. doi: 10.21037/tlcr.2018.09.22 the Lung Immune Prognostic Index With Immune Checkpoint Inhibitor Outcomes in Patients With Advanced Non-Small Cell Lung Cancer. JAMA Oncol 2018;4:351-7. 